\title{
Surgical management of gingival recession using autogenous soft tissue grafts
}

\author{
Peter Windisch $^{1}$ (D) $\cdot$ Balint Molnar ${ }^{1}$ (D)
}

Received: 16 July 2019 / Accepted: 23 September 2019 / Published online: 30 October 2019

(C) The Author(s) 2019

\begin{abstract}
One of the chief goals of periodontal plastic surgery is establishment of ideal pink esthetics through the reconstruction of gingival recessions. A gold standard treatment approach for coverage of gingival recession with predictable esthetic outcomes is the transplantation of autogenous soft tissue grafts. Various surgical techniques can be used in combination with autogenous soft tissue grafts for gingival recession coverage.
\end{abstract}

Keywords Gingival recession · Root coverage $\cdot$ Connective tissue graft · Free gingival graft

\section{Quick reference/description}

One of the chief goals of periodontal plastic surgery is establishment of ideal pink esthetics through the reconstruction of gingival recessions. A gold standard treatment approach for coverage of gingival recession with predictable esthetic outcomes is the transplantation of autogenous soft tissue grafts. Various surgical techniques can be used in combination with autogenous soft tissue grafts for gingival recession coverage.

Peter Windisch

peter.windisch@gmail.com

1 Department of Periodontology, Semmelweis University, Szentkiralyi u. 47, Budapest 1088, Hungary 


\title{
Overview
}

\author{
Surgical approaches Indications Applications \\ for gingival reces- \\ sion coverage using \\ autogenous soft tissue \\ grafts
}

Surgical techniques in combination with free gingival graft (FGG) and partly-epithelialized free gingival graft (PE-FGG)

One-stage FGG/PE- Coverage of single or multiple gingival This technique increases the width of FGG technique

recession defects

attached gingiva for root coverage

Augmentation of the keratinized

gingiva, predominantly in the lateral

zone

Two-stage FGG

technique

Coverage of single or multiple gingival recession

Gingival augmentation is achieved in the first-stage surgery and coronal repositioning of the integrated graft in the second-stage surgery

Surgical techniques used in combination with subepithelial connective tissue graft (SCTG) and epithelialized-subepithelial connective tissue graft (ESCTG) for single gingival recession coverage

Coronally advanced Complete coverage of single Miller's flap technique class I, II gingival recession defects Partial coverage of single Miller's class III gingival recession defects

Semilunar coronally advanced flap technique

Single or multiple gingival recessions with a minimum width of $3 \mathrm{~mm}$ and thickness of $1 \mathrm{~mm}$ of the keratinized gingiva apically from the defect

It is only indicated in cases with a thick gingival biotype and favorable baseline mucogingival conditions

Envelope technique Miller's class I, II, III gingival recessions
Coronally advanced flap approach is the most predictable technique for single gingival recession coverage

It was initially described as a cosmetic periodontics-coronally repositioned pedicle graft

SCAF procedure is an alternative to the CAF technique for long-term tissue stability and superior esthetic results

ET is a feasible approach for the treatment of sites with a shallow vestibular fold like the anterior mandible

Surgical techniques used in combination with SCTG and partly epithelialized soft tissue graft for multiple gingival recession coverage
Modified coronally advanced flap technique
Coverage of multiple gingival reces- sions

Subperiosteal envelope technique

Coverage of multiple adjacent gingival recessions

\section{Modified coronally advanced tunnel technique}

Mainly indicated for perfect color matching and avoidance of scar lines, when used in combination with SCTG for gingival recession coverage in esthetically demanding areas
The MCAF approach is a redesigned version of the classic CAF technique for multiple gingival recession coverage

SET is a modification of the envelope technique and results in uneventful healing with minimal post-operative complications due to lack of blood supply compromise to the papillae

Provides predictable root coverage in multiple Miller's class I, II, III gingival recession defects 


\section{Materials/instruments}

- Resorbable sutures

- Non-resorbable sutures

- No. $15 / 15 C$ surgical blade

- Microsurgical blades

- Tunneling knives

- Gracey curettes

- Blunt elevators

- Microsurgical elevators

\section{Procedure}

Recreation of optimal pink esthetics is the ultimate goal of periodontal plastic surgery. This can be achieved by reconstructing the existing gingival recessions. Autogenous soft tissue grafts are considered as a gold standard treatment approach for gingival recession coverage with predictable tissue stability and esthetics. These grafts can be applied in combination with several different surgical techniques for coverage of gingival recession.

Autogenous free soft tissue grafts are harvested from a remote and esthetically irrelevant region of the oral mucosa and are entirely detached from the donor site. This is useful in avoiding donor site complications surrounding the adjacent teeth. Therefore, minimal risk of impaired esthetics and root hypersensitivity is present because of wound healing via secondary intention at the adjacent sites. However, free autogenous soft tissue grafts application needs a second surgical site with a risk for possible complications like infection, pain, swelling and necrosis that cannot be completely eliminated even through meticulous treatment planning and good surgical skills.

\section{Autogenous soft tissue grafts}

The various autogenous soft tissue grafts that are used for gingival recession coverage are:

\section{Free gingival graft}

Nabers first used the term free gingival graft (FGG). Autogenous grafts are mainly harvested from the palate. FGG is nowadays less frequently used for the treatment of gingival recessions. It has high predictability of post-operative tissue stability and graft survival. Following transplantation to the recipient site, maintenance of the original tissue characteristics by the palatal soft tissue grafts with epithelial coverage is of immense clinical importance. Use of FGG induces favorable amount of keratinization, although with impaired esthetics due to differences in surface color and texture compared to adjacent sites. 


\section{Subepithelial connective tissue graft}

The first grafting approach of choice for root coverage is subepithelial connective tissue graft (SCTG). In periodontal plastic surgery, one of the most esthetically predictable and versatile grafting procedures is transplantation of SCTG. For coverage of denuded root surfaces using the bilaminar reconstruction of lost gingival tissues, SCTG can be applied in conjunction with split-thickness pedicle-, tunneltype or envelope flaps. SCTG application results in enhanced root coverage due to optimal blood supply. Excellent predictability and esthetics may be obtained with SCTG.

In case a change in gingival tissue surface characteristics is required or a significant increase in the width and thickness of keratinized gingiva is essential, SCTGs are not considered as the first grafts of choice, instead, FGG, PE-FGG or ESCTG may be applied. In cases with thin palatal masticatory mucosa and reduced amount of donor tissues, allogeneic and xenogeneic grafts should be considered instead of harvesting connective tissue from the hard palate.

The advantages of SCTG are:

- Enhanced color match and improved esthetic results as the surface characteristics of the overlying flap are similar to that of the adjacent recipient gingiva.

- Wound healing in the donor and recipient sites mostly occurs by primary intention when SCTG is covered by coronally advanced- or tunneled flaps via partialthickness flap preparation.

- This healing by primary intention can facilitate tissue maturation and decrease postoperative discomfort

\section{Partly epithelialized soft tissue grafts}

Epithelialized-subepithelial connective tissue graft (ESCTG) combined with an envelope type flap is a treatment alternative combining the advantages of FGGs and SCTGs. In this technique, the epithelialized grafts are placed to cover the exposed root surfaces. Another grafting procedure is the partly epithelialized free gingival graft (PE-FGG) that is used for gingival recessions of the anterior mandible in conjunction with an apically repositioned flap. Both these grafting approaches provide:

- Increased resistance against the tension of the muscular-mucosal environment.

- Lowering the risk for displacement of the mucogingival junction (MGJ) or flattening of the vestibule.

- Increased amount of keratinized tissues.

- Acceptable color blending compared to FGG. 


\section{Surgical techniques in combination with free autogenous soft tissue grafts}

For the treatment of single and multiple gingival recessions, various techniques in combination with free autogenous soft tissue grafts are available. Several other approaches among the available techniques have been proven to be less techniquesensitive and achieve more predictable esthetic results.

\section{Surgical techniques in combination with FGG and PE-FGG}

One-stage FGG/PE-FGG technique FGGs in conjunction with an apically repositioned flap can be used for increasing the width of attached gingiva. This technique can be used to treat single or multiple gingival recession defects for root coverage and augmentation of the keratinized gingiva, predominantly in the lateral zone. As per the standard apically repositioned flap approach, the one-stage FGG/PE-FGG technique is performed as follows (Fig. 1).

- A split-thickness flap is prepared following root planning. A 3-5 mm wide zone of the superficial layer of the flap is removed for preparing a recipient periosteal bed.

- The hard palate or the maxillary tuberosity is usually used for harvesting the FGG.

- The harvested FGG is adapted carefully to the recipient periosteum and the adjacent gingiva using resorbable or non-resorbable $6 / 0$ monofilament sutures.

- Pressure is exerted for 1-2 min on the surgical site to avoid graft necrosis due to blood clots between the two layers.

Graft surface desquamation and subsequent re-epithelialization from the adjacent sites occurs if the wound healing is uneventful with no inflammation. As an alternative to the use of FGG, a PE-FGG can be utilized with the same surgical technique to deliver similar tissue stability and enhanced color blending.

(a)

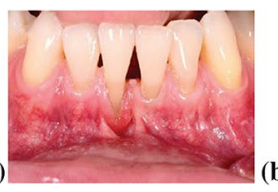

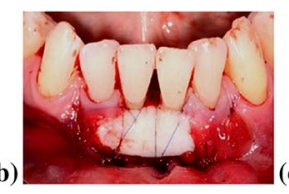

(c)

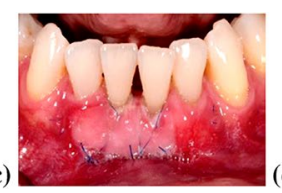

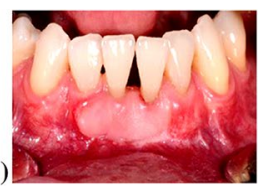

Fig. 1 Apically repositioned flap in combination with a free gingival graft for treatment of multiple Miller class IV defects. a Baseline, b graft in place, c 14 days healing, $\mathbf{d} 1$ year outcome 
Two-stage FGG technique The two-stage FGG technique includes a first-stage surgery for gingival augmentation and a second-stage surgery for coronal repositioning of the integrated graft. The first-stage surgery is performed like the one-stage FGG/PE-FGG technique. The main distinguishable feature of this approach from the classical apically repositioned flap-FGG technique is the preservation of pre-existing keratinized gingiva. An FGG is adapted to the periosteal bed apically to widen the residual keratinized gingiva around the recession defects after an incision is placed at the MGJ. A coronally advanced flap is elevated to reposition the previously expanded zone of keratinized tissues for root coverage after a minimum of 2 months of graft integration.

\section{Surgical techniques used in combination with SCTGs and ESCTGs for single recession coverage}

Coronally advanced flap technique Brustein first described the coronally advanced flap (CAF) as a cosmetic periodontics-coronally repositioned pedicle graft. Several modifications have been developed. The most predictable technique for coverage of single gingival recessions is the CAF technique. The requirement criteria for success of CAF as a part of the two-stage FGG approach was outline by Maynard in 1977 as:

- Presence of shallow crevicular depths on proximal surfaces

- Anatomical interproximal bone heights

- Tissue height within $1 \mathrm{~mm}$ of the cemento-enamel junction of adjacent teeth

- 6-week healing of a FGG prior to coronal positioning

- Reduction of any root prominence

- Adequate flap release during surgery to prevent retraction during healing

The coronally advanced flap approach is as follows (Fig. 2).

- Outline of the surgical site is achieved with a bilateral trapezoid incision on the medial and distal aspects of the exposed root surface. Using No. 15C sur-

(a)

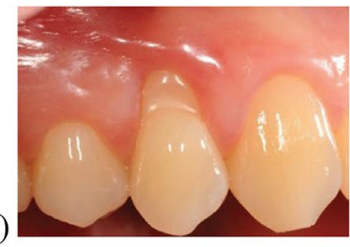

(b)

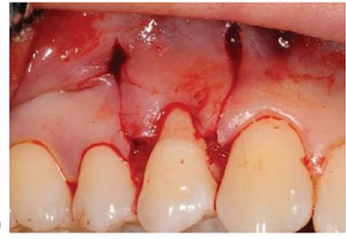

(c)

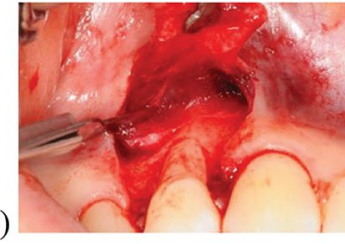

(d)

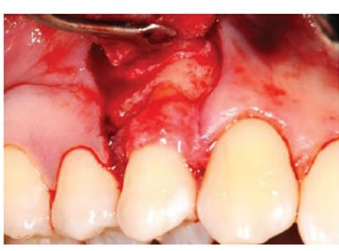

(e)

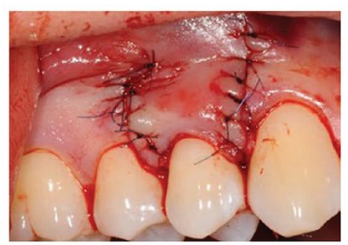

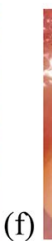

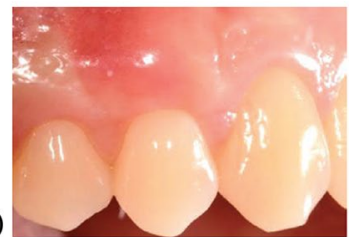

Fig. 2 Coronally advanced flap with a SCTG for treatment of single Miller class I defect. a Baseline b incisions c split-full-split preparation d SCTG application e Sutures $\mathbf{f} 5$ years outcome 
gical blade or microsurgical blades, bilateral horizontal split-thickness incisions are made at a distance from the tip of the anatomical papilla to level of $1 \mathrm{~mm}$ more than the recession depth.

- Following the trapezoid incisions, diverging vertical releasing incisions are placed.

- At the papillary zone, a split-thickness flap is prepared. The split-thickness flap is then followed by elevation of the full-thickness of attached gingiva (from gingival zenith to MGJ) using blunt elevators.

- Split-thickness flap elevation is continued by sharp dissection from the MGJ into the vestibule by detachment of the loose and flexible mucosal-submucosal layers from the underlying muscles and periosteum.

- A harvested SCTG is placed at the level of the CEJ after complete flap mobilization.

- The graft is fixed with mattress sutures to the adjacent mucosa or resorbable sutures to the recipient periosteal bed-complete de-epithelialization of the anatomical papillae is done.

- The flap is coronally advanced for full coverage of the de-epithelialized papillae.

- Double-sling non-resorbable 6/0 monofilament sutures are used to secure the flap margin $1 \mathrm{~mm}$ coronally from the CEJ.

- Closure of the vertical incisions is achieved using diagonally placed single interrupted sutures, beginning from the most apical point.

- Pressure is exerted for 1-2 min on the surgical site to avoid graft necrosis.

- Sutures are removed after 14 days.

Current standards suggest that complete root coverage is possible in Miller class I-II cases and partial coverage can be expected in Miller class III cases using the CAF procedure.

Semilunar coronally advanced flap technique For the treatment of single or multiple recessions with a minimum width of $3 \mathrm{~mm}$ and thickness of $1 \mathrm{~mm}$ of the keratinized gingiva apically from the defect, an alternative to the CAF approach is the semilunar coronally advanced flap (SCAF) procedure. It is only indicated in cases with a thick gingival biotype and favorable baseline mucogingival conditions without additional free soft tissue grafts. Long-term tissue stability and superior esthetic results are achieved with the SCAF technique. It is performed as follows (Fig. 3): 
(a)

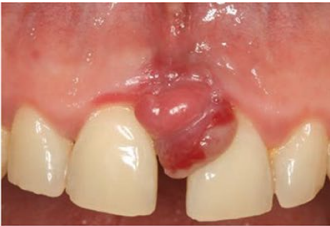

(d)

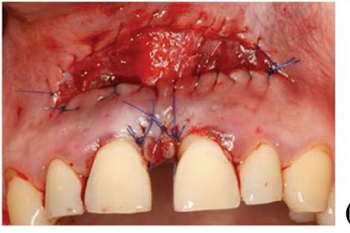

(b)
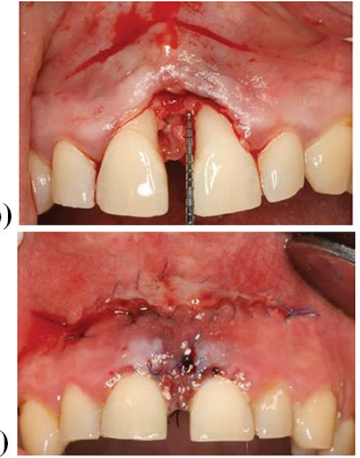

(c)

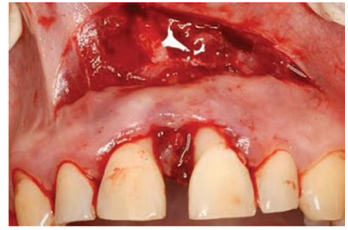

(f)

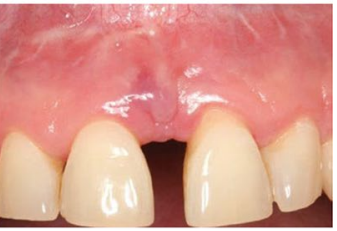

Fig. 3 SCAF technique for the removal of parodontoma gigantocellulare. a Baseline $\mathbf{b}$ after excision $\mathbf{c}$ semilunar flap elevation $\mathbf{d}$ sutures $\mathbf{e} 7$ days healing $\mathbf{f} 2$ years outcome

- Local anesthesia is administered.

- At the level of the MGJ, a semilunar incision is made.

- A split-thickness flap is elevated beginning from the sulcus.

- Using 6/0 non-resorbable monofilament sutures, the keratinized gingival collar is mobilized and secured at the level of the CEJ. The sutures are removed after 14 days.

Envelope technique The envelope technique (ET) is used in conjunction with SCTGs or ESCTGs to cover Miller class I, II, and III gingival recessions. The lack of coronal flap advancement is a chief advantage of this approach. Therefore, the ET is considered feasible for the treatment of sites with a shallow vestibular fold like the anterior mandible. The steps of ET are (Fig. 4).

(a)

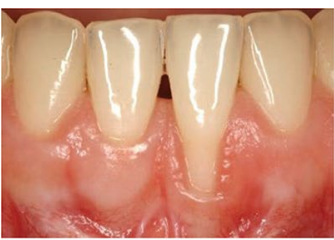

(d)

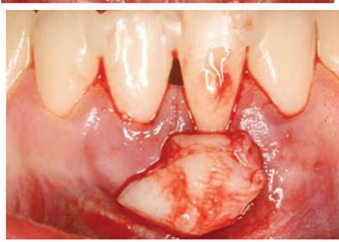

(b)
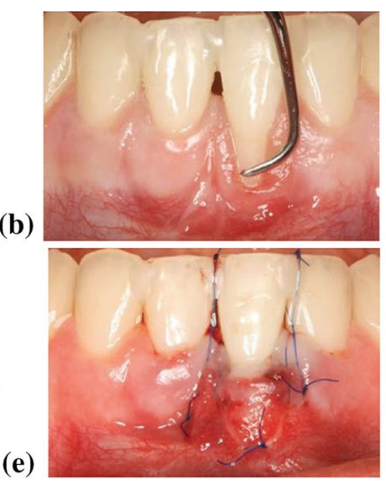

(c)

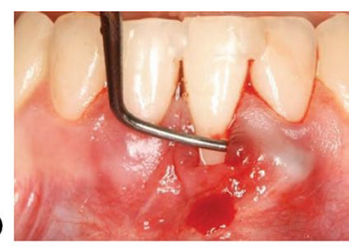

(f)

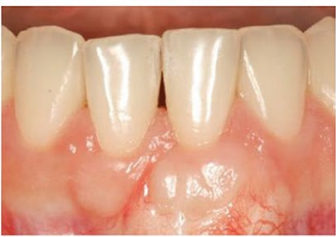

Fig. 4 Envelope technique with an ESCTG for treatment of a single Miller class III defect. a Baseline b root planing, $\mathbf{c}$ envelope preparation d ESCTG application e sutures $\mathbf{f} 4$ years outcome 
- A sharp dissection using a $15 \mathrm{C}$ surgical blade or microsurgical blades is performed commencing from the sulcus towards the adjacent papillae to prepare a split-thickness envelope flap (gingival pouch). The depth of the flap is dependent on the dimensions of the previously harvested graft.

- A SCTG or an ESCTG is inserted into the envelope at the level of the CEJ.

- 5/0 or 6/0 non-resorbable, mono-filament, single interrupted, mattress or sling sutures are used for graft and flap fixation. The sutures are removed after 14 days.

\section{Surgical techniques used in combination with SCTGs and ESCTGs for multiple recession coverage}

Modified coronally advanced flap technique For coverage of multiple recessions, the modified coronally advanced flap (MCAF) technique is used (Fig. 5). It is a redesigned version of the classic CAF approach. The MCAF also involves the use of:

- Split-thickness preparation of the interdental papilla

- Full-thickness preparation of the keratinized gingiva between the gingival zenith and the MGJ

- Split-thickness preparation of the mucosal flap beyond MGJ

The main difference from the original CAF technique is in the releasing incisions that outline the surgical papillae. The direction of the oblique incisions is always towards the center of the flap. The flap center in most cases is a canine or a midline papilla that is tunneled. This is then followed by graft placement and fixation with sutures. The sutures are removed after 14 days.

(a)

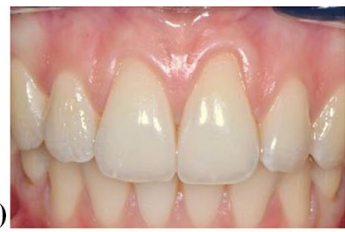

(d)

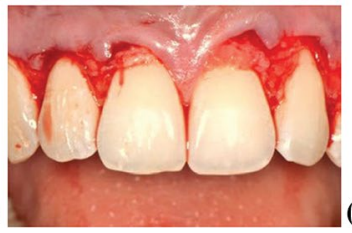

(b)

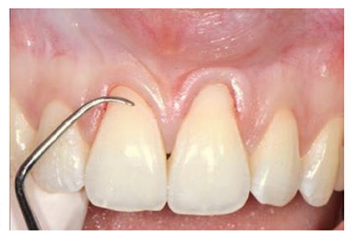

(c)

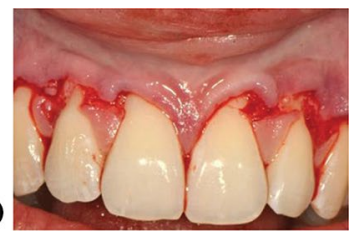

(e)

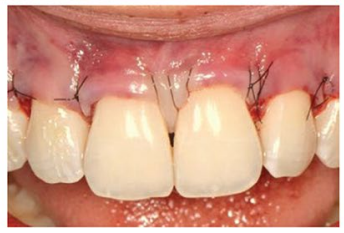

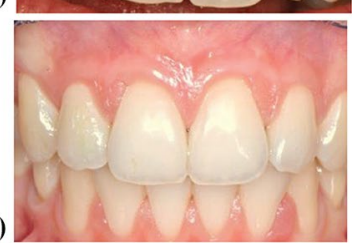

Fig. 5 Modified coronally advanced flap (MCAF) with a SCTG for treatment of multiple Miller class I defects. a Baseline b incisions $\mathbf{c}$ split-full-split preparation $\mathbf{d}$ SCTG application e sutures $\mathbf{f} 1$ year outcome (Courtesy of Dr. Ferenc Bartha and Dr. Dóra Kovács) 
Subperiosteal envelope technique A modification of the original envelope technique for the management of multiple adjacent gingival recessions is the subperiosteal envelope technique (SET). A major advantage of the SET is that there is no compromise of blood circulation of the papillae resulting in uneventful healing and minimal post-surgical complaints. The SET approach results in enlargement of the keratinized zone with excellent color blending of the graft and recipient site owing to the secondary epithelialization of the placed grafts. The SET is performed as follows (Fig. 6).

- Local anesthesia is administered.

- The exposed root surfaces are planed after local anesthesia administration.

- Microsurgical or $15 \mathrm{C}$ surgical blade is used to place intrasulcular incisions around the involved teeth.

- Elevation of the mucoperiosteal envelope flap is done by blunt preparation up to the level of the MGJ at each individual recession site using tunneling knives without involving the tips of the interdental papillae.

- A confluent tunnel is prepared over all the exposed root surfaces by interconnecting the separate mucoperiosteal envelopes.

- The tunnel preparation is followed by preparation of the MGJ in a split thickness of up to 3-5 $\mathrm{mm}$ depth.

- A harvested SCTG or ESCTG is then adapted to the created supraperiosteal envelope using horizontal mattress sutures. The graft can also be secured by sling sutures. The sutures are removed after 14 days.

(a)

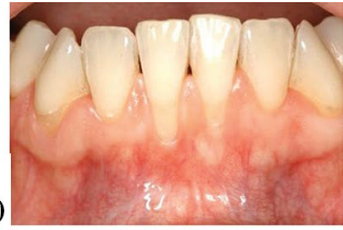

(d)

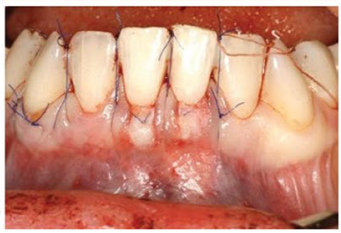

(b)

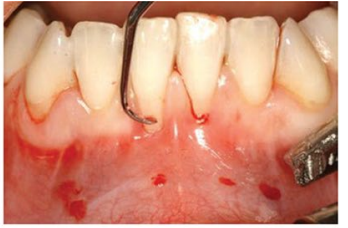

(c)

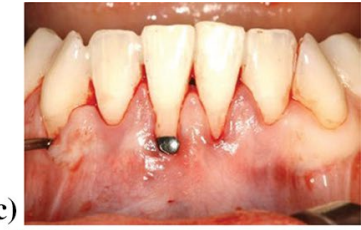

(e)

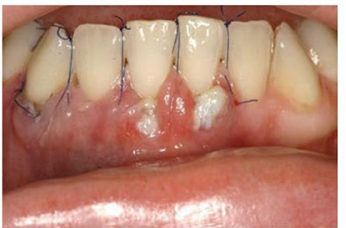

(f)

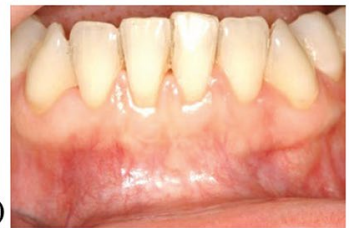

Fig. 6 Subperiosteal envelope technique for treatment of multiple Miller class III defects. a Baseline b root planing $\mathbf{c}$ tunneling $\mathbf{d}$ sutures placed with partially exposed graft $\mathbf{e} 7$ days healing $\mathbf{f} 1$ year outcome 
Modified coronally advanced tunnel technique The modified coronally advanced tunnel technique (MCAT) is an adaptation of the original SET that allows predictable root coverage, even in multiple Miller class III recessions. The MCAT technique is mainly indicated to achieve a perfect color blending and no scar lines at the treated sites, when used in combination with SCTG. The MCAT involves (Fig. 7).

- More excessive split-thickness flap mobilization

- Separation and release of inserting collagen fibers and attaching muscles from the inner aspect of the alveolar mucosa via Gracey curettes and tunneling knifes. Therefore, allowing extensive mobilization of the tunneled flap and tensionless coronal advancement.

- Complete flap mobilization is achieved by careful and gentle undermining of the interdental papillae with microsurgical elevators. Disruption of the interdental papillae should be avoided.

- SCTG fixation to the mucosal flap is achieved using horizontal mattress sutures after positioning the coronal margin of the graft at the level of the CEJ.

- Coronal advancement of the flap is done using suspended or sling sutures after securing the graft in the tunnel.

- To allow suspended suturing, preoperative resin bonding of adjacent contact points can be performed at the surgical site. Sling sutures are placed interdentally to facilitate coronal displacement of the tunnel $1 \mathrm{~mm}$ over the CEJ when complete coverage of the graft cannot be obtained with the suspended sutures. The sutures are removed after 14 days.

(a)

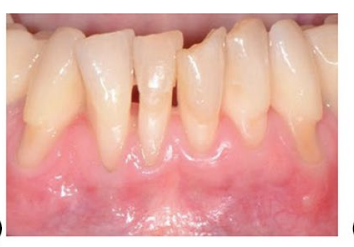

(b)

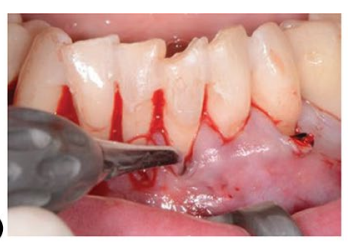

(c)

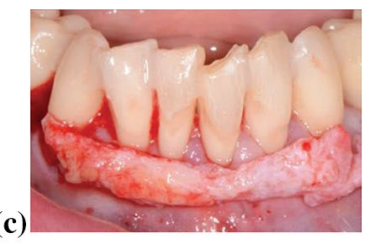

(d)

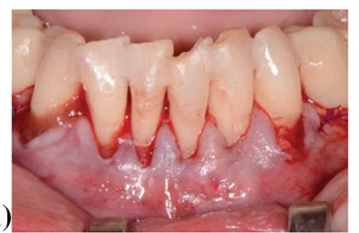

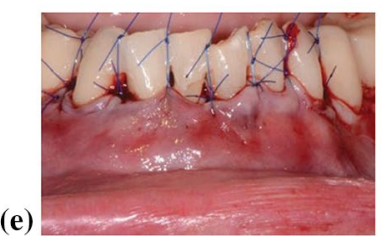

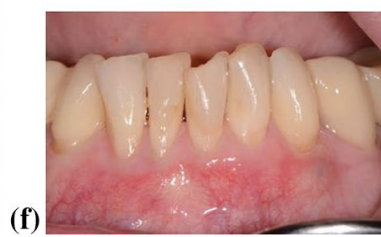

Fig. 7 MCAT with SCTG for treatment of multiple Miller class III defects. a Baseline b Tunneling c Trimmed SCTG d SCTG in the tunnel, e suspended sutures $\mathbf{f} 2$ years outcome 


\section{Pitfalls and complications}

- Over-augmentation of tissue contours due to graft hyperplasia and impaired color blending between the graft and recipient site are the major common complications associated with FGG use. Therefore, FGG is not considered to be a valid treatment alternative in esthetically demanding situations.

- Following the one-stage FGG/PE-FGG technique of gingival recession coverage, graft loosening or infection can occur and lead to graft necrosis or partial recession.

- A chief limitation of the CAF technique is the lack of keratinized gingiva.

- After soft tissue grafting with the subperiosteal envelope technique, epithelial invagination and cicatrization at the borders of the exposed root surfaces can result in scar lines.

Acknowledgements Open access funding provided by Semmelweis University (SE). This work was supported by the Hungarian Human Resources Development Operational Program (EFOP-3.6.2-16-2017-00006).

Open Access This article is distributed under the terms of the Creative Commons Attribution 4.0 International License (http://creativecommons.org/licenses/by/4.0/), which permits unrestricted use, distribution, and reproduction in any medium, provided you give appropriate credit to the original author(s) and the source, provide a link to the Creative Commons license, and indicate if changes were made.

\section{Further reading}

1. Windisch P, Molnár B (2018) Recession coverage using autogenous grafts. In: Kasaj A (ed) Gingival recession management. Springer, Cham. https://doi.org/10.1007/978-3-319-70719-8_8

2. Carnio J (2014) Modified apically repositioned flap technique: a surgical approach to enhance donor sites prior to employing a laterally positioned flap. Int J Periodontics Restorative Dent. 34(3):423-429

3. Cairo F, Pagliaro U, Buti J, Baccini M, Graziani F, Tonelli P, Pagavino G, Tonetti MS (2016) Root coverage procedures improve patient aesthetics. A systematic review and Bayesian network metaanalysis. J Clin Periodontol. 43(11):965-975

4. Tatakis DN, Chambrone L, Allen EP, Langer B, McGuire MK, Richardson CR, Zabalegui I, Zadeh HH (2015) Periodontal soft tissue root coverage procedures: a consensus report from the AAP Regeneration Workshop. J Periodontol 86(2 Suppl):S52-S55

5. Tonetti MS, Jepsen S, Working Group 2 of the European Workshop on Periodontology (2014) Clinical efficacy of periodontal plastic surgery procedures: consensus report of Group 2 of the 10th European Workshop on Periodontology. J Clin Periodontol. 41(Suppl 15):S36-S43

6. Cairo F, Nieri M, Pagliaro U (2014) Efficacy of periodontal plastic surgery procedures in the treatment of localized facial gingival recessions. A systematic review. J Clin Periodontol. 41(Suppl 15):S44-S62

7. Bernimoulin JP, Lüscher B, Mühlemann HR (1975) Coronally repositioned periodontal flap. Clinical evaluation after 1 year. J Clin Periodontol. 2(1):1-13

8. Zucchelli G, De Sanctis M (2000) Treatment of multiple recession-type defects in patients with esthetic demands. J Periodontol 71(9):1506-1514

Publisher's Note Springer Nature remains neutral with regard to jurisdictional claims in published maps and institutional affiliations. 